\title{
PROCEEDINGS THE ROYAL

\section{The reinfection threshold promotes variability in tuberculosis epidemiology and vaccine efficacy}

M. Gabriela M. Gomes, Ana O. Franco, Manuel C. Gomes and Graham F. Medley

Proc. R. Soc. Lond. B 2004 271, 617-623

doi: $10.1098 /$ rspb.2003.2606

\section{References}

Email alerting service
Article cited in:

http://rspb.royalsocietypublishing.org/content/271/1539/617\#related-urls

Receive free email alerts when new articles cite this article - sign up in the box at the top right-hand corner of the article or click here 


\title{
The reinfection threshold promotes variability in tuberculosis epidemiology and vaccine efficacy
}

\author{
M. Gabriela M. Gomes ${ }^{1 *}$, Ana O. Franco ${ }^{1}$, Manuel C. Gomes ${ }^{2}$ \\ and Graham F. Medley ${ }^{3}$ \\ ${ }^{1}$ Instituto Gulbenkian de Ciência, Apartado 14, 2781-901 Oeiras, Portugal \\ ${ }^{2}$ Departamento de Biologia Vegetal E CMAF, Faculdade de Ciencias de Lisboa, Bloco C2, Piso 4, Campo Grande, \\ 1749-016 Lisboa, Portugal \\ ${ }^{3}$ Ecology and Epidemiology Group, Department of Biological Sciences, University of Warwick, Coventry CV4 7AL, UK
}

\begin{abstract}
Population patterns of infection are determined largely by susceptibility to infection. Infection and vaccination induce an immune response that, typically, reduces susceptibility to subsequent infections. With a general epidemic model, we detect a 'reinfection threshold', above which reinfection is the principal type of transmission and, consequently, infection levels are much higher and vaccination fails. The model is further developed to address human tuberculosis (TB) and the impact of vaccination. The bacille Calmette-Guérin (BCG) is the only vaccine in current use against $\mathrm{TB}$, and there is no consensus about its usefulness. Estimates of protection range from 0 to $80 \%$, and this variability is aggravated by an association between low vaccine efficacy and high prevalence of the disease. We propose an explanation based on three postulates: (i) the potential for transmission varies between populations, owing to differences in socio-economic and environmental factors; (ii) exposure to mycobacteria induces an immune response that is partially protective against reinfection; and (iii) this protection is not significantly improved by BCG vaccination. These postulates combine to reproduce the observed trends, and this is attributed to a reinfection threshold intrinsic to the transmission dynamics. Finally, we demonstrate how reinfection thresholds can be manipulated by vaccination programmes, suggesting that they have a potentially powerful role in global control.
\end{abstract}

Keywords: tuberculosis; vaccine efficacy; epidemiological model; partial immunity; reinfection threshold

\section{INTRODUCTION}

The invasion threshold for infectious pathogens has been a keystone concept for many decades (Kermack \& McKendrick 1927; Diekmann et al. 1990). Simply stated, if the average number of susceptible individuals infected by a single infected individual is greater than one, then the infection prevalence will increase. In a naive population, susceptibility is at its maximum. Infection itself and vaccination both reduce susceptibility to infection and disease. The simplest assumption is that the immune response to primary infection provides a solid immunity to subsequent infections or reinfections, and this gives rise to the susceptible-infectedrecovered (SIR) and susceptible-exposed-infectedrecovered (SEIR) frameworks (Anderson \& May 1991).

The development of ideas about partial susceptibility (or partial immune protection) has been driven largely by considerations of interactions between various multistrain pathogens (White et al. 1998; Ferguson \& Andreasen 2002; Gomes et al. 2002) and of vaccine-escape mutants (McLean 1995). Here, we extend these ideas to consider the role of reinfection susceptibility in determining the prevalence of infection. We develop a simple SIR model where the recovered are partially susceptible to reinfection. We demonstrate that there is a reinfection threshold above which the prevalence of infection reaches much

*Author for correspondence (ggomes@igc.gulbenkian.pt). higher levels. If a vaccine does not confer complete protection against reinfection, then it can interact with the transmission dynamics to produce considerable variability in vaccine outcome.

We apply the ideas to human tuberculosis (TB) and vaccination with bacille Calmette-Guérin (BCG). Field trials to evaluate BCG efficacy began in the 1930s, and by the late 1950s the range of estimates was broad. Subsequent trials have continued to record variable results (Fine \& Rodrigues 1990; Bloom \& Fine 1994; Colditz et al. 1994). Initial explanations of the observed variability have invoked differences between vaccine strains, trial methodology, host genetics and socio-economic conditions. Another proposed explanation is that regional differences in environmental mycobacteria (EM) might be responsible for much of the observed variability (Palmer \& Long 1966; Fine 1995). The reasoning is that exposure to EM imparts some degree of protection against Mycobacterium tuberculosis (Mtb), which is not improved by the BCG vaccination, leading to an apparent absence of protection by the vaccine when trials are conducted in areas of high EM exposure. In a sense, exposure to EM is acting as a vaccination. This interference has been experimentally demonstrated by studies in animal models (Palmer \& Long 1966; Edwards et al. 1982; Brandt et al. 2002) and, more recently, by immuno-epidemiological field studies in human populations (Black et al. 2002).

Geographical trends support the suggested influence of EM on the outcome of vaccination. Namely, estimates of BCG efficacy appear to increase with latitude, and 
geographical patterns of EM reveal higher prevalences at lower latitudes (Palmer \& Long 1966; Fine 1995). However, this argument does not explain the higher prevalence of disease found at lower latitudes, just where immunity induced by EM should be more common. How does $M t b$ maintain high prevalence in those regions where the majority of the population is 'vaccinated' by exposure to EM?

A complementary observation is that immunity induced either by a previous infection or by vaccine uptake does not fully prevent reinfection (ten Dam \& Pio 1982; van Rie et al. 1999; Godfrey-Faussett et al. 2000; Smith et al. 2000). In this scenario, both the observed prevalence of disease and the impact of vaccination programmes must depend on the dynamic balance between primary infection and reinfection rates, which, in turn, depend on all socioeconomic and environmental factors that affect transmission (Lienhardt 2001).

We construct a simple mathematical model that reproduces the described trends. The model encapsulates three postulates: (i) the potential for transmission varies between populations; (ii) exposure to mycobacteria induces an immune response that is partially protective against reinfection; and (iii) this protection is not significantly improved by vaccination.

\section{BASIC MODEL STRUCTURE AND ANALYSIS}

Before describing the dynamics of TB transmission we extract some background results from a general SIR framework, where those who have recovered from a primary infection or have been vaccinated have reduced susceptibility to infection:

$$
\begin{aligned}
& \frac{\mathrm{d} S}{\mathrm{~d} t}=(1-v) \mu-\beta I S-\mu S, \\
& \frac{\mathrm{d} I}{\mathrm{~d} t}=\beta I(S+\sigma R)-(\tau+\mu) I, \\
& \frac{\mathrm{d} R}{\mathrm{~d} t}=v \mu+\tau I-\sigma \beta I R-\mu R .
\end{aligned}
$$

The variables $S, I$ and $R$ are the proportions of the population susceptible, infected and recovered (with partial immune protection), respectively; the life expectancy of hosts at birth is $1 / \mu$; the average duration of infectiousness is $1 / \tau$, and $\beta$ is the transmission coefficient. The parameter $\sigma(0 \leqslant \sigma \leqslant 1)$ determines the degree of partial protection given by a primary infection or vaccination: $\sigma=0$ implies complete protection (i.e. the classic SIR model), and $\sigma=1$ implies no protection (i.e. the susceptible-infectedsusceptible (SIS) model). The parameter $v$ represents the proportion of the population vaccinated at birth. The model is built under the assumption that the mode of action of acquired immunity is independent of whether stimulation comes from infection or vaccination.

Without vaccination $(v=0)$, the basic reproduction number is $R_{0}=\beta /(\tau+\mu)$ (Diekmann et al. 1990). Algebraic manipulation shows that the model has a single stable non-trivial equilibrium above a first (invasion) threshold $\left(R_{0}=1\right)$. At equilibrium, we have $\mathrm{S}+$ $\sigma R=1 / R_{0}$, and the prevalence of infection $(I)$ is highly sensitive to a second (reinfection) threshold $\left(R_{0}=1 / \sigma\right)$. When $1<R_{0}<1 / \sigma$, most infections occur in naive susceptibles $(S)$, but when $R_{0}>1 / \sigma$, most infections occur in the previously infected $(R)$ class, as this is generally larger and

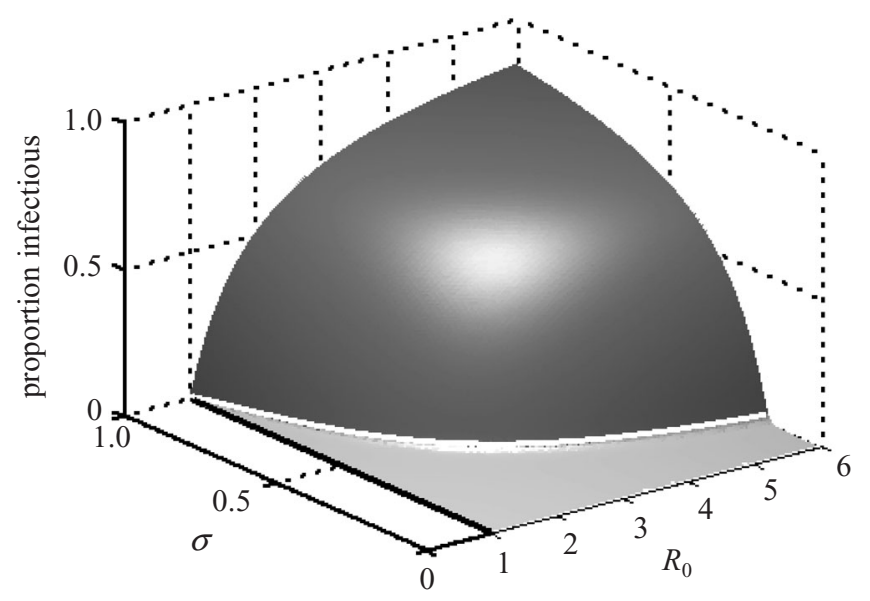

Figure 1. Two thresholds of transmission obtained from model (2.1). The black line $\left(R_{0}=1\right)$ represents the invasion threshold, above which infection persists in the host population. The white line $\left(R_{0}=1 / \sigma\right)$ represents the reinfection threshold, where the prevalence of infection rises steeply to much higher levels. The surface represents the proportion of individuals that are infectious at equilibrium, and this is coloured light grey below the reinfection threshold, and dark grey above.

the infection potential is great enough to overcome the immunity given by previous infection. Another view is that $R_{0}$ determines the model behaviour: for $1<R_{0}<1 / \sigma$ the model acts much as an SIR framework, and for $R_{0}>1 / \sigma$ the model demonstrates typical SIS behaviour. The two thresholds are determined by the two parameters $R_{0}$ and $\sigma$, as shown in figure 1: the black line represents the invasion threshold, and the white line represents the reinfection threshold. Below the invasion threshold, infection does not persist, and above it there are two possibilities. Either the proportion of infected individuals tends to a 'low endemic equilibrium' (light-grey surface), or the level of infection stabilizes at a 'high endemic equilibrium' (dark-grey surface). Model (2.1) has been thoroughly analysed (Gomes et al. (2004b), where the impact of vaccination is also examined). Similar thresholds were obtained in the context of multi-strain dynamics (Gomes et al. 2002, fig. $3 a$ ) where the 'reinfection threshold' gave rise to oscillatory dynamics. Without further assumptions, the modelled vaccine can have very high efficacy below the reinfection threshold and virtually no efficacy above it. This is highly suggestive of what is happening with pulmonary TB.

\section{TUBERCULOSIS MODEL STRUCTURE}

A model for TB epidemiology must differentiate individuals according to their history of infection by $M t b$. We start by specifying four categories: $S$, susceptible individuals, who have never encountered the mycobacterium; I, infected individuals, who have active disease and are infectious; L, latent individuals, who are infected but have not developed active disease and are not infectious; and R, recovered or cured individuals, who were previously infected and have lost that infection. According to previous estimates (Vynnycky \& Fine 1997), a proportion $\phi=0.1$ of infections progress to active TB, while the remaining 0.9 maintain a latent infection. Latent and recovered individuals have acquired some immunity, which reduces the risk of subsequent infection, but does 
(a)

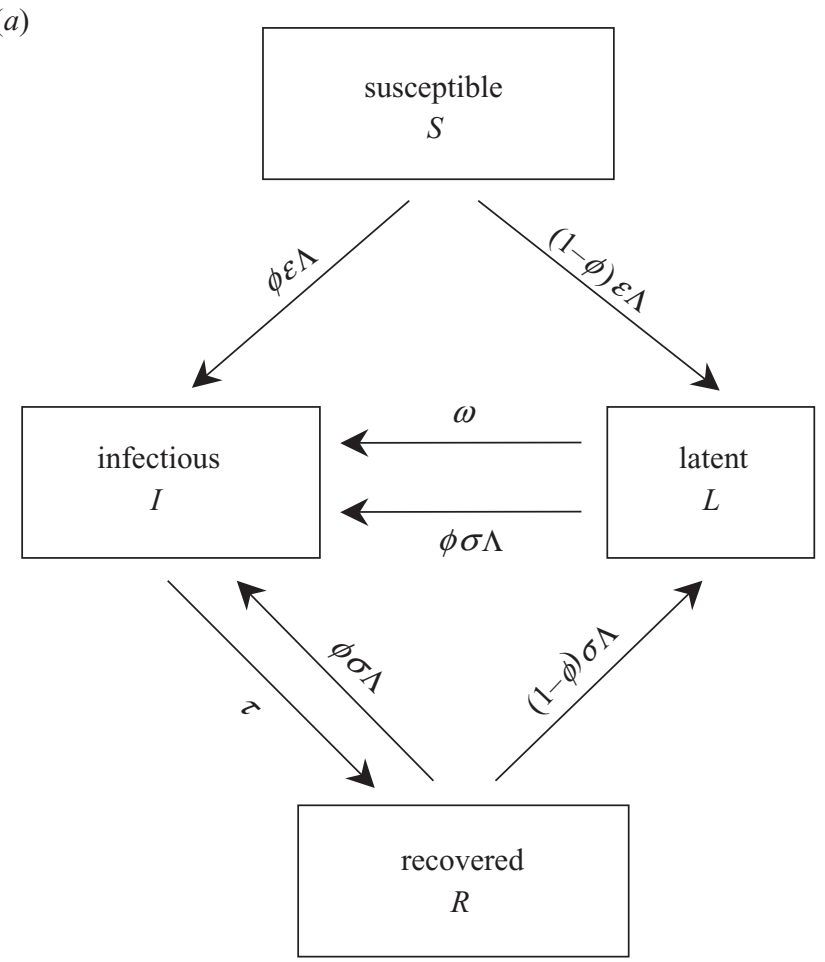

(b)

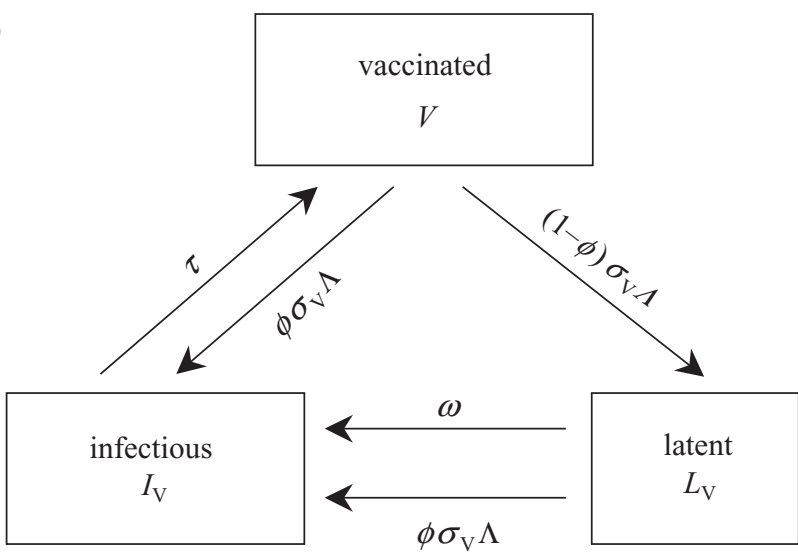

Figure 2. Model for TB transmission dynamics. (a) Nonvaccinated subpopulation. (b) Vaccinated subpopulation. All parameters, except $\varepsilon$ and $\Lambda$, are described in table 1 . The auxiliary parameter, $\varepsilon$, is 1 by default and this is changed only to generate sub-models in figure 3 .

not fully prevent it. Latent TB can progress to active TB by either exogenous reinfection or endogenous reactivation (at a rate $\omega$ ). Recovered individuals can revert to active TB by exogenous reinfection only. The dynamics of infection, immunity and treatment are described by the diagram in figure $2 a$ and formalized by the system of differential equations

$$
\begin{aligned}
& \frac{\mathrm{d} S}{\mathrm{~d} t}=(1-v) \mu-\varepsilon \Lambda S-\mu S, \\
& \frac{\mathrm{d} I}{\mathrm{~d} t}=\phi \Lambda(\varepsilon S+\sigma(R+L))+\omega L-(\tau+\mu) I, \\
& \frac{\mathrm{d} L}{\mathrm{~d} t}=(1-\phi) \Lambda(\varepsilon S+\sigma R)-\phi \sigma \Lambda L-(\omega+\mu) L, \\
& \frac{\mathrm{d} R}{\mathrm{~d} t}=\tau I-(\sigma \Lambda+\mu) R .
\end{aligned}
$$

These equations represent the rates of change of each category and are expressed in terms of the parameters described in table 1. Extra notations are $\varepsilon$, the auxiliary parameter, which is 1 by default and permits the generation of sub-models in figure 3 , and $\Lambda$, the per capita rate of infection, which is discussed further below. The model is extended to incorporate vaccination at birth. This requires the further specification of three categories: V, vaccinated individuals who are not infected; and vaccinated individuals who have been infected and are either infectious with active TB $\left(\mathrm{I}_{\mathrm{V}}\right)$ or latent $\left(\mathrm{L}_{\mathrm{V}}\right)$. Transmission in the vaccinated population is described in figure $2 b$ and, using the parameters in table 1 , this is formalized as

$$
\begin{aligned}
& \frac{\mathrm{d} V}{\mathrm{~d} t}=v \mu+\tau I_{\mathrm{V}}-\sigma_{\mathrm{V}} \Lambda V-\mu V, \\
& \frac{\mathrm{d} I_{\mathrm{V}}}{\mathrm{d} t}=\phi \sigma_{\mathrm{V}} \Lambda\left(V+L_{\mathrm{V}}\right)+\omega L_{\mathrm{V}}-(\tau+\mu) I_{\mathrm{V}}, \\
& \frac{\mathrm{d} L_{\mathrm{V}}}{\mathrm{d} t}=(1-\phi) \sigma_{\mathrm{V}} \Lambda V-\phi \sigma_{\mathrm{V}} \Lambda L_{\mathrm{V}}-(\omega+\mu) L_{\mathrm{V}} .
\end{aligned}
$$

Three parameters require special attention. The first refers to the rate of infection, $\Lambda$, of a naive individual. We make the usual assumption (Kermack \& McKendrick 1927; Anderson \& May 1991) that this rate is proportional to the density of infectious individuals, $\Lambda=\beta\left(I+I_{\mathrm{V}}\right)$. The constant of proportionality is the parameter $\beta$, known as the 'transmission coefficient'. This parameter summarizes all socio-economic and environmental factors that affect transmission, and its variability reflects postulate (i). The second refers to the rate of reinfection, $\sigma \Lambda$, of an individual who has previously been infected and has acquired some degree of protective immunity. The parameter $\sigma$ is the factor reducing the risk of reinfection, and this is central to postulate (ii). For the purposes of illustration, we fix $\sigma=0.25$, but any value between 0 and 1 would lead to the same conclusions. The third parameter, $\sigma_{\mathrm{V}}$, is the factor by which vaccination reduces the risk of reinfection, and is initially assumed to be equal to $\sigma$, so fulfilling postulate (iii). The parameter $\sigma_{\mathrm{V}}$ will be subsequently lowered to illustrate the effect of vaccines that induce more protection than does the infection itself. Some authors state that the principal effect of BCG is to reduce disease progression rather than the risk of actual infection (Smith et al. 2000). This would correspond to introducing $\sigma_{\mathrm{V}}$ as a factor of $\phi$ (and perhaps also $\omega$ ) rather than as a factor of $\Lambda$. Our results extend to these alternative assumptions.

\section{TUBERCULOSIS RESULTS}

Figure 3 describes the proportion of individuals with active TB at equilibrium, in the absence of vaccination. The full curve corresponds to the full model, and the others result from two sub-models illustrating the contributions of primary infection (dotted line) and reinfection (dashed line). Applying standard methodology (Diekmann et al. 1990) the basic reproduction number associated with model (3.1) is calculated as

$R_{0}=\frac{(\omega+\phi \mu) \beta}{(\omega+\mu)(\tau+\mu)}$

and, in analogy with the basic SIR model described in $\int 2$, the two thresholds for transmission are 
Table 1. Model parameters.

\begin{tabular}{lll}
\hline symbol & definition & value \\
\hline$\mu$ & death rate and birth rate & $1 / 70 \mathrm{yr}^{-1}$ \\
$\phi$ & proportion of individuals that develop active TB. The remaining $1-\phi$ have latent $\mathrm{TB}$ & 0.1 \\
$\sigma$ & $\begin{array}{l}\text { factor reducing the risk of infection as a result of acquired immunity to a previous } \\
\quad \text { infection }\end{array}$ & 0.25 \\
& factor reducing the risk of infection as a result of vaccination & $0.25 ; 0.20$ \\
$\sigma_{\mathrm{V}}$ & vaccination coverage & $95 \%$ \\
$\omega$ & rate of endogenous reactivation of latent TB & $0.0002 \mathrm{yr}^{-1}$ \\
$\tau$ & rate of treatment of active TB & $2 \mathrm{yr}^{-1}$ \\
$\beta$ & transmission coefficient & variable
\end{tabular}

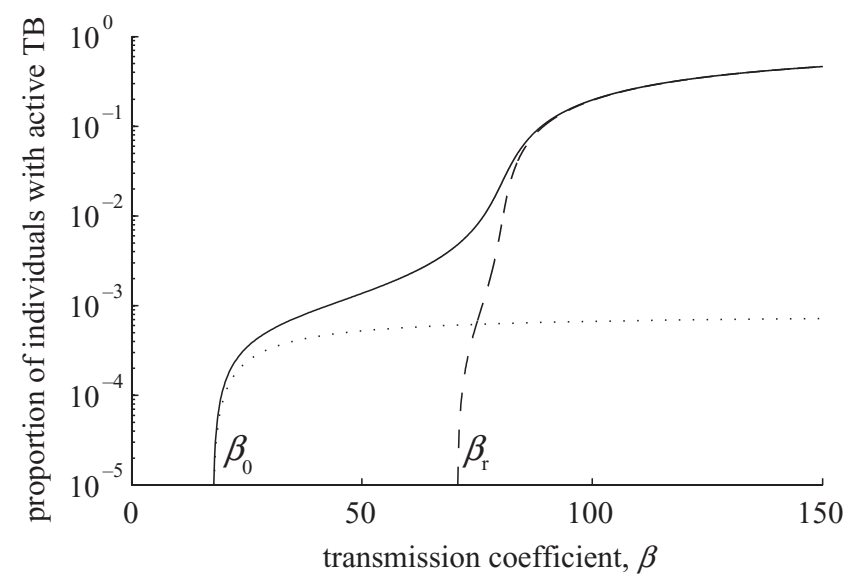

Figure 3. Two thresholds in TB transmission, in the absence of vaccination. The full line represents the equilibrium proportion of individuals with active TB as a function of the transmission coefficient. The dotted line represents the contribution of primary infection (model (3.1) with $\sigma=0$ ) and is associated with the invasion threshold, $\beta_{0}=(\tau+\mu)$ $(\omega+\mu) /(\omega+\phi \mu)$. The dashed line outlines the contribution of reinfection (model (3.1) with $\varepsilon=\sigma=0.25$ ) and is used to mark the reinfection threshold, $\beta_{\mathrm{r}}=\beta_{0} / \sigma$. These two processes are nonlinearly coupled in the full model (model (3.1) with $\varepsilon=1$ and $\sigma=0.25$ ) to produce the full curve. $\omega=0.0002$.

invasion threshold $\left(R_{0}=1\right): \quad \beta_{0}=\frac{(\omega+\mu)(\tau+\mu)}{\omega+\phi \mu}$,

reinfection threshold $\left(R_{0}=1 / \sigma\right): \beta_{\mathrm{r}}=\frac{(\omega+\mu)(\tau+\mu)}{(\omega+\phi \mu) \sigma}$.

The invasion threshold indicates the minimum transmission potential required for persistence of the infection in the population, and the reinfection threshold is the transmission potential above which reinfection dominates the dynamics. For transmission coefficients between $\beta_{0}$ and $\beta_{r}$, the majority of disease results from primary infection. For transmission coefficients greater than $\beta_{\mathrm{r}}$, high exposure overcomes protective immunity and the majority of disease is caused by reinfection. As a result, the prevalence of disease is relatively low for $\beta_{0}<\beta<\beta_{\mathrm{r}}$, rising by two orders of magnitude near $\beta_{\mathrm{r}}$ to remain high thereafter. A similar bimodal structure was recently demonstrated in hepatitis B epidemiology as a result of a different mechanism (Medley et al. 2001).
Figure 4 shows the effect of a vaccination programme: $95 \%$ coverage with a vaccine that protects as much as a previous infection $\left(\sigma_{\mathrm{V}}=\sigma\right)$. The full curves in figure $4 a$ show the proportions of individuals with active TB at equilibrium: the higher curve refers to the absence of vaccination, as in figure 3, and the lower curve corresponds to the presence of vaccination. The three dots (marked A, $B, C)$ represent model populations with different transmission coefficients, used to simulate the impact of vaccination over time (figure $4 b$ ). The proportion of cases prevented by the vaccination programme is shown in figure $4 c$. The variability in vaccination impact is evident: it ranges from highly effective at low prevalences (population A) to having a negligible effect at high prevalences (population C). A vaccine that protects only as well as a previous infection will have no practical effect unless the transmission coefficient is below the reinfection threshold, $\beta_{\mathrm{r}}$. Similarly, interference with EM would have no noticeable impact on populations whose transmission coefficients are above $\beta_{\mathrm{r}}$, but it could contribute to a significant reduction in TB incidence in populations with lower transmission coefficients.

So far we have assumed that infection and vaccination induce equivalent immune responses but this is unlikely to be exactly so. If the vaccine reduces susceptibility by a factor $\sigma_{\mathrm{V}} \neq \sigma$, then the transmission threshold for vaccine protection is $\beta_{\mathrm{rV}}=\left(\sigma / \sigma_{\mathrm{V}}\right) \beta_{\mathrm{r}}$. A vaccine with a reduced efficacy $\left(\sigma_{\mathrm{V}}>\sigma\right)$ fails to be protective when the transmission coefficient is above $\beta_{\mathrm{rV}}$, which is lower than $\beta_{\mathrm{r}}$. For a reasonable range of $\sigma_{\mathrm{V}}$ values, the overall impact of such a vaccine would be similar to that represented in figure 4 . In particular, the variable efficacy discussed for $\sigma_{\mathrm{V}}=\sigma$ extends to the case $\sigma_{\mathrm{V}}>\sigma$. This is generally accepted as the BCG scenario.

The most exciting and optimistic results are those for a vaccine efficacy greater than the protection induced by infection $\left(\sigma_{\mathrm{V}}<\sigma\right)$. Such a vaccine would increase the reinfection threshold and consequently enlarge the range of populations where vaccination can reach high efficacy (see figure 5). This is most evident for population $\mathrm{C}$, where vaccines discussed previously $\left(\sigma_{\mathrm{V}} \geqslant \sigma\right)$ registered efficacies close to nil, and in which the new vaccine can reduce the prevalence of active $\mathrm{TB}$ by more than two orders of magnitude. The reinfection threshold can be manipulated by appropriate vaccines and, if carefully quantified, it can play a key role in setting targets for future vaccine performance and use. 

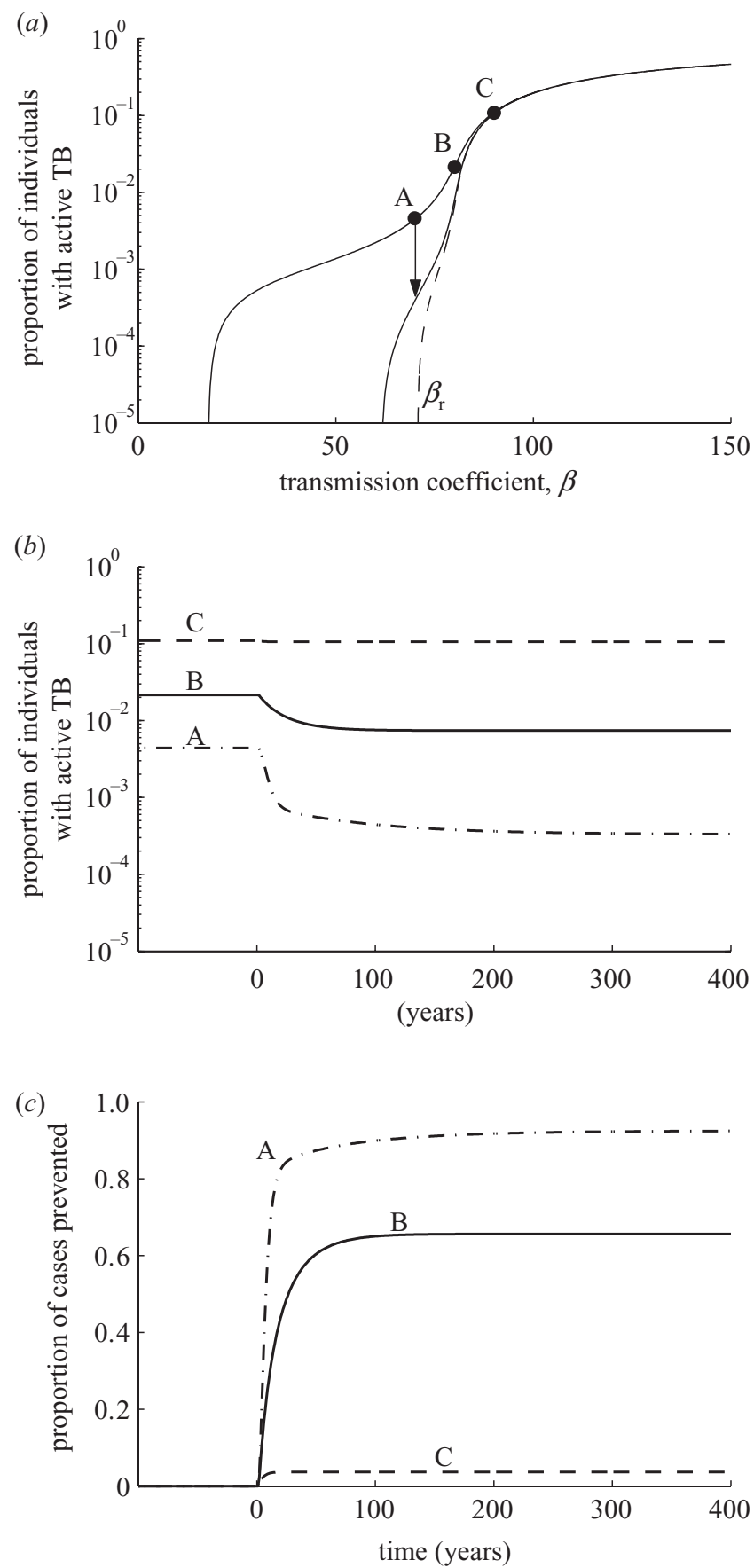

Figure 4. The impact of vaccination, given that the protective effect of the vaccine does not exceed that of acquired immunity to a previous infection $\left(\sigma_{\mathrm{V}}=\sigma\right)$. (a) Proportions of individuals with active TB at equilibrium, before and after implementation of a mass vaccination programme with $95 \%$ coverage. The dashed line represents the ideal scenario of $100 \%$ vaccination coverage, and is equivalent to that obtained from the reinfection sub-model in figure 3. The three dots (marked A, B, C) illustrate the pre-vaccination equilibria of three model populations $\left(\sigma_{\mathrm{v}}=0.25\right)$. (b) Simulation of the effects of mass vaccination on the three model populations. The prevalence of disease, $I(t)+I_{\mathrm{V}}(t)$, gradually decreases towards the post-vaccination equilibrium. (c) Proportion of cases prevented by the vaccination programme, $1-\left(I(t)+I_{\mathrm{V}}(t)\right) /\left(I(0)+I_{\mathrm{V}}(0)\right)$, as a function of time. Time is measured in years, and the beginning of vaccination is set at $t=0$.

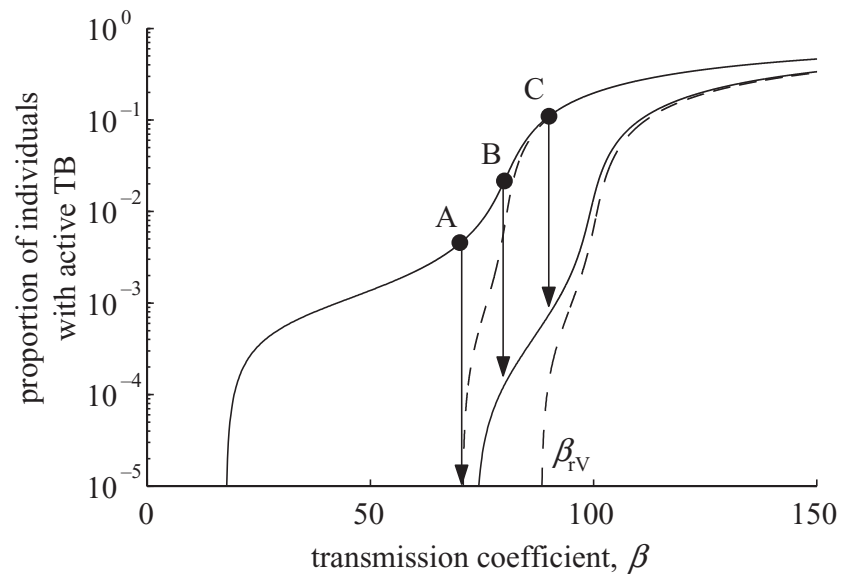

Figure 5. Impact of a vaccine that is more potent than a previous infection $\left(\sigma_{\mathrm{v}}<\sigma\right)$. The solid curves represent the proportion of individuals with active TB before (higher curve) and after (lower curve) the implementation of a vaccination programme. The vaccination coverage is $95 \%$ and the susceptibility reduction factor is $\sigma_{\mathrm{V}}=0.20$. The dashed lines represent the ideal scenario of $100 \%$ coverage: the lower curve corresponds to $\sigma_{\mathrm{v}}=0.20$, while the higher corresponds to $\sigma_{\mathrm{V}}=0.25$ as in figure 4 , and is repeated here for comparison.

\section{DISCUSSION}

Using a minimal model for transmission dynamics, we have demonstrated that three well-accepted postulates generate the dominant trends in TB epidemiology. To our knowledge, this is the first model to explain, simultaneously, the apparent variabilities in BCG vaccination efficacy and TB prevalence. Furthermore, we demonstrate that the two variabilities act synergistically, and are therefore inseparable. We have shown how reinfection can be a major promoter of variability in TB incidence, and how this interferes with an individual's response to vaccination. Although the variabilities are promoted by nonlinearities in the population dynamics of infection and reinfection, the predicted variable efficacy of the vaccine will be apparent in individual measurements. For example, BCG is expected to confer more protection to an individual in the UK than to an individual in a highly endemic region, because exposure to $M t b$ interferes with BCG and reduces its efficacy (Smith et al. 2000). This fundamental aspect of TB epidemiology has not been captured by previous models, as they have not fully implemented reinfection (Blower et al. 1995, 1996; Dye et al. 1998; Dye \& Williams 2000) or vaccination (Vynnycky \& Fine 1997).

The model was then used to discuss prospects for control. At this stage, agreement between model output and epidemiological data is restricted to broad trends. Enhanced accuracy requires the incorporation of further complexities into the model, and the treatment of heterogeneities in field data and trial design (Sutherland et al. 1982; Styblo 1991; Comstock 1994). In particular, measures of immune protection (here represented by $\sigma$ and $\sigma_{\mathrm{v}}$ ) are crucial players and deserve special care. Effective immunity results from the interaction of various mechanisms (Smith et al. 1984). It has been debated whether immunity confers some protection to all individuals, reducing their susceptibility by a factor $\sigma$ ('partial' immunity), or immunity confers full protection to some 
individuals, leaving a fraction $\sigma$ unprotected ('all-ornothing' immunity). It is also argued that immunity induced by the BCG vaccine wanes over time ('temporary' immunity). The real scenario is likely to combine the three mechanisms, and the development of models that consider the dynamics of immunity more deeply can be illuminating. In the meantime, we have demonstrated that partial protection promotes variability, and this should extend to more complex mechanisms of immunity that involve partial protection.

TB is widespread and requires global control measures. As past empirical experience has shown, and the present theory explains, a major difficulty lies in achieving a significant impact in those regions where the disease prevalence is high owing to reinfection $\left(\beta>\beta_{\mathrm{r}}\right)$. Reducing transmission can have a significant impact on TB prevalence, especially if it is brought below the reinfection threshold. This is probably what the developed world experienced from the mid-1800s until the 1970s, owing to major improvements in socio-economic conditions. However, in less developed countries this is a difficult and slow process. The development of more potent vaccines against TB is a hopeful possibility (Britton \& Palendira 2003; Olsen \& Andersen 2003; Reed et al. 2003). In the meantime, the most immediate measure to keep TB under control is the rapid detection and treatment of active TB cases (Dye et al. 1998; Dye \& Williams 2000; World Health Organization 2002). A recent extension to this approach is to identify and treat persons with latent infection to prevent the development of active disease. This substantial shift in management is vigorously implemented in the USA (Small \& Fujiwara 2001), and its extension to other regions of the world is being debated (Chaisson 2000). Again, variable outcomes emerge as a result of variable reinfection rates (Gomes et al. 2004a). The success of detect-and-treat programmes will be determined largely by characteristics of the target population and by the comparative susceptibility of those who are latent and those who have recovered.

In conclusion, understanding reinfection is fundamental to understanding TB epidemiology, to exploring the available options for control and to guiding the development of new interventional tools. These conclusions are not only relevant to TB but more generally applicable to diseases characterized by recurrence of infection. The reinfection threshold is a concept introduced here to represent the transmissibility required to promote recurrent infections. Populations that exceed this threshold sustain high levels of infection and tend to be insensitive to interventions. Finally, and most excitingly, reinfection thresholds can be manipulated by interventions, providing new hope for the control of infectious disease in high-burden regions.

The authors thank António Coutinho, Isabel Gordo and Leonor Parreira for valuable comments on previous versions of this manuscript. This research was initiated while M.G.M.G. was a Wellcome Trust Research Fellow at the University of Warwick, and continued with funding from The Gulbenkian Foundation and FCT of Portugal.

\section{REFERENCES}

Anderson, R. M. \& May, R. M. 1991 Infectious diseases of humans: dynamics and control. Oxford University Press.
Black, G. F. (and 16 others) 2002 BCG-induced increase in interferon-gamma response to mycobacterial antigens and efficacy of BCG vaccination in Malawi and the UK: two randomised controlled studies. Lancet 359, 1393-1401.

Bloom, B. R. \& Fine, P. E. 1994 The BCG experience: implications for future vaccines against TB. In Tuberculosis: pathogenesis, protection and control (ed. B. R. Bloom), pp. 531-552. Washington, DC: American Society for Microbiology.

Blower, S. M., McLean, A. R., Porco, T. C., Small, P. M., Hopewell, P. C., Sanchez, M. A. \& Moss, A. R. 1995 The intrinsic transmission dynamics of tuberculosis epidemics. Nature Med. 1, 815-821.

Blower, S. M., Small, P. M. \& Hopewell, P. C. 1996 Control strategies for tuberculosis epidemics. Science 273, 497-500.

Brandt, L., Cunha, J. F., Olsen, A. W., Chilima, B., Hirsch, P., Appleberg, R. \& Andersen, P. 2002 Failure of the Mycobacterium bovis BCG vaccine: some species of environmental mycobacteria block multiplication of BCG and induction of protective immunity to tuberculosis. Infect. Immun. 70, 672-678.

Britton, W. J. \& Palendira, U. 2003 Improving vaccines against tuberculosis. Immunol. Cell Biol. 81, 34-45.

Chaisson, R. E. 2000 New developments in the treatment of latent tuberculosis. Int. f. Tuberc. Lung Dis. 4(Suppl. 2), S176-S181.

Colditz, G. A., Brewer, T. F., Berkey, C. S., Wilson, M. E., Burdick, E., Fineberg, H. V. \& Mosteller, F. 1994 Efficacy of BCG vaccine in the prevention of tuberculosis. $7 A M A$ 271, 698-702.

Comstock, G. W. 1994 Field trials of tuberculosis vaccines: how could we have done them better? Control. Clin. Trials 15, 247-276.

Diekmann, O., Heesterbeek, J. A. P. \& Metz, J. A. J. 1990 On the definition and the computation of the reproduction number $R_{0}$ in models for infectious diseases in heterogeneous populations. F. Math. Biol. 28, 365-382.

Dye, C. \& Williams, B. G. 2000 Criteria for the control of drug-resistant tuberculosis. Proc. Natl Acad. Sci. USA 97, 8180-8185.

Dye, C., Garnett, G. P., Sleeman, K. \& Williams, B. G. 1998 Prospects for worldwide tuberculosis control under the WHO DOTS strategy. Lancet 352, 1886-1891.

Edwards, M. L., Goodrich, J. M., Muller, D., Pollack, A., Ziegler, J. E. \& Smith, D. W. 1982 Infection with Mycobacterium avium-intracellulare and the protective effect of bacille Calmette-Guérin. F. Infect. Dis. 145, 733-741.

Ferguson, N. \& Andreasen, V. 2002 The influence of different forms of cross-protective immunity on the population dynamics of antigenically diverse pathogens. In Mathematical approaches for emerging and reemerging infectious diseases (ed. S. Blower, C. Castillo-Chavez, K. L. Cooke, D. Kirschner \& P. Van der Driessche), pp. 157-170. IMA Volumes in Mathematics and its Applications. New York: Springer.

Fine, P. E. M. 1995 Variation in protection by BCG: implications of and for heterologous immunity. Lancet 346, 1339-1345.

Fine, P. E. M. \& Rodrigues, L. C. 1990 Modern vaccines: mycobacterial diseases. Lancet 335, 1016-1020.

Godfrey-Faussett, P., Sonnenberg, P., Shearer, S. C., Bruce, M. C., Mee, C., Morris, L. \& Murray, J. 2000 Tuberculosis control and molecular epidemiology in a South African goldmining community. Lancet 356, 1066-1071.

Gomes, M. G. M., Medley, G. F. \& Nokes, D. J. 2002 On the determinants of population structure in antigenetically diverse pathogens. Proc. R. Soc. Lond. B 269, 227-233. (DOI 10.1098/rspb.2001.1869.)

Gomes, M. G. M., Gomes M. C. \& Medley, G. F. $2004 a$ Treatment of latent tuberculosis in light of the reinfection threshold. Proc. R. Soc. Lond. B. (Submitted.) 
Gomes, M. G. M., White, L. J. \& Medley, G. F. $2004 b$ Infection, reinfection and vaccination under suboptimal immune protection: epidemiological perspectives. F. Theor. Biol. (In the press.)

Kermack, W. O. \& McKendrick, A. G. 1927 A contribution to the mathematical theory of epidemics. Proc. R. Soc. Lond. A $115,700-721$.

Lienhardt, C. 2001 From exposure to disease: the role of environmental factors in susceptibility to and development of tuberculosis. Epidemiol. Rev. 23, 288-301.

McLean, A. R. 1995 Vaccination, evolution and changes in the efficacy of vaccines: a theoretical framework. Proc. R. Soc. Lond. B 261, 389-393.

Medley, G. F., Lindop, N. A., Edmunds, W. J. \& Nokes, D. J. 2001 Hepatitis-B virus endemicity: heterogeneity, catastrophic dynamics and control. Nature Med. 7, 619-624.

Olsen, A. W. \& Andersen, P. 2003 A novel TB vaccine; strategies to combat a complex pathogen. Immunol. Lett. 85, 207-211.

Palmer, C. E. \& Long, M. W. 1966 Effects of infection with atypical mycobacteria on BCG vaccination and tuberculosis. Am. Rev. Respir. Dis. 94, 553-568.

Reed, S. G., Alderson, M. R., Dalemans, W., Lobet, Y. \& Skeiky, Y. A. W. 2003 Prospects for a better vaccine against tuberculosis. Tuberculosis 83, 213-219.

Small, P. M. \& Fujiwara, P. I. 2001 Management of tuberculosis in the United States. New Eng. F. Med. 345, 189-200.

Smith, D., Wiegeshaus, E. \& Balasubramanian, V. 2000 An analysis of some hypotheses related to the Chingelput bacille
Calmette-Guérin trial. Clin. Infect. Dis. 31(Suppl. 3), S77S80.

Smith, P. G., Rodrigues, L. C. \& Fine, P. E. M. 1984 Assessment of the protective efficacy of vaccines against common diseases using case-control and cohort studies. Int. F. Epidemiol. 13, 87-93.

Styblo, K. 1991 Epidemiology of tuberculosis. Selected papers. The Hague: KNCV.

Sutherland, I., Švandová, E. \& Radhakrishna, S. E. 1982 The development of clinical tuberculosis following infection with tubercle bacilli. Tubercle 62, 255-268.

ten Dam, H. \& Pio, A. 1982 Hypothesis: pathogenesis of tuberculosis and effectiveness of BCG vaccination. Tubercle 63, 225-253.

van Rie, A., Warren, R., Richardson, M., Victor, T. C., Gie, R. P., Enarson, D. A., Beyers, N. \& van Helden, P. D. 1999 Exogenous reinfection as a cause of recurrent tuberculosis after curative treatment. New Eng. F. Med. 341, 1174-1179.

Vynnycky, E. \& Fine, P. E. M. 1997 The natural history of tuberculosis: the implications of age-dependent risks of disease and the role of reinfection. Epidemiol. Infect. 119, 183-201.

White, L. J., Cox, M. J. \& Medley, G. F. 1998 Cross immunity and vaccination against multiple microparasite strains. IMA f. Math. Appl. Med. Biol. 15, 211-233.

World Health Organization 2002 Global tuberculosis control. Geneva: WHO.

As this paper exceeds the maximum length normally permitted, the authors have agreed to contribute to production costs. 